\title{
MicroRNA-373-3p inhibits the growth of cervical cancer by targeting AKT1 both in vitro and in vivo
}

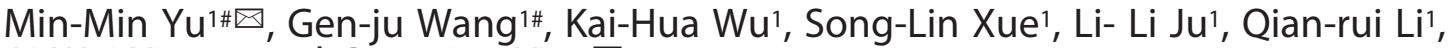 \\ Ai-Wei Xiong ${ }^{1}$ and Guo-ping Yin ${ }^{2} \square$
}

1Department of Obstetrics and Gynecology, The Second Hospital of Nanjing, Nanjing University of Chinese Medicine, Nanjing, 210003, China; 2Department of Anesthesiology, The Second Hospital of Nanjing, Nanjing University of Chinese Medicine, Nanjing, 210003, China

\begin{abstract}
Objective: In this study, we aimed to investigate the function of microRNA-373-3p (miR-373-3p) in the pathogenesis of cervical cancer. Methods: Human and mouse cervical cancer cell lines were transfected with miR-373$3 p$ mimic and inhibitor. Cell proliferation and viability were evaluated with Cell Counting Kit-8 (CCK-8) assay and Lactate Dehydrogenase (LDH) assay, respectively. The AKT1-targeting role of miR-373-3p was analyzed by qPCR and Western blot. Finally, a mouse xenograft cervical tumor model was adopted to study the in vivo effect of miR-373-3p on tumor growth and the expression of AKT1. Results: Over-expression of miR-373-3p significantly reduced the proliferation of cervical carcinoma cell line in vitro. In addition, miR-373-3p overexpression also inhibited cervical cancer growth in tumor-bearing mice. Mechanistically, we found that AKT1 gene can be targeted by miR-373-3p. MiR-373-3p mimic decreased the mRNA and protein expression of AKT1, while the miR-373-3p inhibitor increased the level of AKT1 in cervical cancer cells. AKT1 overexpression rescued the proliferation of cervical cancer cells transfected with miR-3733p. Conclusion: MiR-373-3p can serve as a novel anti-tumor microRNA in cervical cancer by targeting AKT1.
\end{abstract}

Key words: microRNA-373-3p, AKT1, cervical cancer, cell proliferation

Received: 07 August, 2020; revised: 11 November, 2020; accepted: 08 January, 2021; available on-line: 08 July, 2021

『e-mail: XCoip45@163.com (M-M Y); njyy041@njucm.edu.cn (G-p Y) Acknowledgements of Financial Support: Project supported by the National Natural Science Foundation of China (Grant No. 81472431) and Jiangsu Provincial Medical Talent (No. ZDRCA2016072)

"Min-Min Yu and Gen-ju Wang contributed equally to this research. Abbreviations: CSCC, cervical squamous cell carcinoma; LUAD, lung adenocarcinoma; mTOR, mammalian Target of Rapamycin; NC, negative control; PC, positive control; 3-UTR, 3-untranslated region

\section{INTRODUCTION}

Cervical cancer is the seventh most common malignant tumor, and cervical squamous cell carcinoma (CSCC) is the most common type of cervical cancer (Ngoma et al., 2019). Surgery, radiotherapy, and chemotherapy are the main treatment approaches of CSCC. Although these treatment strategies have made some progress in the past several decades, the survival rate of CSCC patients has not been obviously improved, especially in the middle and late stage CSCC patients. Once being diagnosed, many CSCC patients have already lost the opportunity of radical surgery, prone to resistance to radiotherapy and chemotherapy, and the lack of effec- tive treatment poses a serious threat to women's health. Therefore, identifying new targets for the early diagnosis and treatment of CSCC is of great importance for improving the prognosis of cervical cancer.

MicroRNAs are non-coding RNAs of a length of about 20-25 nucleotides. MiRNAs are degraded or inhibited by the mRNA translation of the target gene by not fully or fully binding to the target-based non-translated region (3-untranslated region, 3-UTR), thereby achieving the post-transcriptional level regulation of the target gene expression (Manikandan et al., 2008). Numerous studies have shown that there is a complex link between the development of the tumor and the miRNAs. MicroRNAs may function as either oncogene or anticancer gene in a different types of cancer. It is found that about $50 \%$ of miRNA genes are located at fragile sites in the genome or in cancer-related regions (Croce et al., 2005). They play important roles in the development, invasion, metastasis and angiogenic processes. Abnormal expression of miRNAs can be seen in many tumors, including cervical cancer, which is involved in the occurrence and progression of tumors. For example, miR-9-5p could promote angiogenesis and radiosensitivity in cervical cancer by targeting SOCS5 (Wei et al., 2019). MicroRNA-432 is downregulated in cervical cancer and directly targets Fibronectin1 to inhibit cell proliferation and invasion (Wang et al., 2019). Downregulation of microRNA-1246 inhibits the growth and promotes the apoptosis of cervical cancer cells by targeting thrombospondin-2 (Du et al., 2019). MiR-338 could inhibit the proliferation and autophagy in cervical cancer cells by targeting ATF2 via mammalian Target of Rapamycin (mTOR) signaling pathway, suggesting the potential application of miR-338 in cervical cancer treatment (Lu et al., 2018). MiR-139-5p was another tumor suppressor in cervical cancer pathogenesis via targeting Transcription Factor 4 (TCF4), thereby inhibiting Wnt/ $\beta$-catenin signaling (Ji et al., 2019).

MiR-373-3p has been shown to regulate the development of some kinds of cancer. For instance, miR373-3p downregulation leads to the increased cell growth in lung adenocarcinoma (LUAD) cells, suggesting that miR-373-3p may act as a potential anticancer strategies in LUAD (Fan et al., 2018). To date, whether miR-373-3p plays a role in modulating the pathogenesis of cervical cancer is still unknown. In this work, we investigated the function of miR-373-3p in cervical cancer using both mouse tumor model and in vitro experiments and identified miR-373-3p as a new anti-tumor microRNA in cervical cancer. Finding the function of characteristic microRNAs in CSCC and related molecular mechanism may provide new 
molecular markers and therapeutic targets for CSCC and be of great importance in the diagnosis and treatment of CSCC. The high expression of miR-21 in cervical cancer and HeLa cell indicates that it may play a possible role of oncogenes, while miR-143 and miR373 with low expression may play the role of tumor suppressor genes (Liu et al., 2012). These works indicate that miR-373 can serve as a tumor suppressor gene. However, the role and mechanism of miR-373 in cervical cancer remains unclear so far. Therefore, in this work, we investigated the role of miR-373-3p in the progression of cervical cancer.

\section{MATERIALS AND METHODS}

\section{Cell culture}

TC-1 cells generated by the co-transformation of C57BL/6 mouse lung epithelial cells with HPV16 E6 and E7 oncogenes and the human ras oncogene were purchased from Chinese Academy of Medical Sciences. Human cervical cancer lines - HeLa (ATCC ${ }^{\circledR}$ CRM-CCL-2TM) and CaSki (ATCC ${ }^{\circledR}$ CRM-CRL-1550) cells, were purchased from American Type Culture Collection. Human cervical cancer cells were cultured in Dulbecco's Modified Eagle Medium (\#PM150210, Procell Life Science\&Technology, China) supplemented with 10\% fetal bovine serum (\#10437-028, Gibco, Carlsbad, CA). TC-1 cells were cultured in RPMI-1640 medium (\#SH3002701, HyClone, USA) supplemented with $10 \%$ fetal bovine serum.

\section{Cell proliferation assay}

3000 cells were seeded in each well and subjected to indicated treatment for $24 \mathrm{~h}$ and $48 \mathrm{~h}$. Afterwards, $90 \mathrm{uL}$ of fresh medium and $10 \mu \mathrm{L}$ of CCK-8 solution (\#CK04, CCK-8, Dojin, Japan) was added to each well. $2.5 \mathrm{~h}$ later, the absorbance was measured at $450 \mathrm{~nm}$ on an Infinite M200 FA plate reader (TECAN, Männedorf, Switzerland).

A

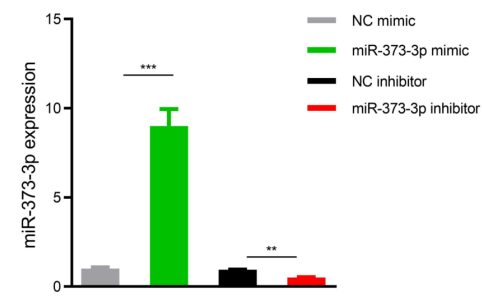

C

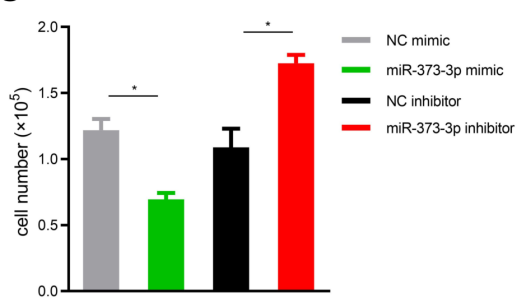

\section{Quantitative real-time PCR (qPCR)}

The cells were homogenized in $1 \mathrm{ml}$ of TRIzol (15596026, Invitrogen, CA) to extract the total RNA following the protocols. The PrimeScript ${ }^{\mathrm{TM}}$ RT Reagent Kit (\#RR037B, TaKaRa, Japan) was used to synthesis cDNA. Q-PCR was performed for AKT1 and GAPDH. The relative expressions were normalized to GAPDH or U6 using the comparative cycle threshold method and fold change. The primers used were as follows: AKT1 forward primer, 5'-AGCGACGTGGCTATTGTGAAG-3'; AK'T1 reverse primer, 5'-GCCATCATTCTTGAGGAGGAAGT-3'; GAPDH forward primer, 5'-GGAGCGAGATCCCTCCAAAAT-3'; GAPDH reverse primer, 5'-GGCTGTTGTCATACTTCTCATGG-3'. miR-373-3p forward primer, 5'-CGGGGAGTAGTATGGAGTCGGCG-3'; miR-373-3p reverse primer, 5'-AACTTCGTCCTCCAAAATCGCCCG-3'; U6 forward primer, 5'-TCGGCAGCACATATACTAA-3'; U6 reverse primer, 5'-ATGGAACGCT'TCACGAAT-3'.

\section{Cytotoxicity assay}

The cytotoxicity of TC-1 cells was analyzed by Lactate dehydrogenase $(\mathrm{LDH})$ release assay using $\mathrm{LDH}$ Cytotoxicity Assay Kit (ab65393, Abcam) following the manufacturer's instructions. Briefly, $3 \times 103$ TC- 1 cells transfected with miR-373-3p mimic, miR-373-3p inhibitor or control were seeded in triplicates in 96well plate and cultured for 48 hours. Wells with medium only (without cells) served as a negative control (NC). $10 \mu \mathrm{L}$ of LDH Reaction Mix was added to each well and incubated for $40 \mathrm{~min}$ at the room temperature. For the positive control (PC), $10 \mu \mathrm{l}$ of Cell Lysis Solution was added before incubation with LDH Reaction Mix. The absorbance was measured at $450 \mathrm{~nm}$ on an Infinite M200 FA plate reader (TECAN, Männedorf, Switzerland). Absorbance at $650 \mathrm{~nm}$ served as the reference wavelength. Cytotoxicity $(\%)=(\mathrm{OD}$ sample - ODNC)/(ODsample - ODPC) $\times 100$.

\section{B}

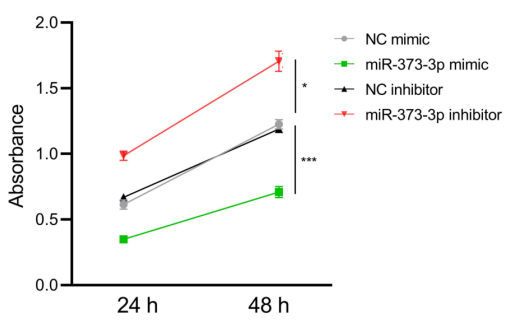

D

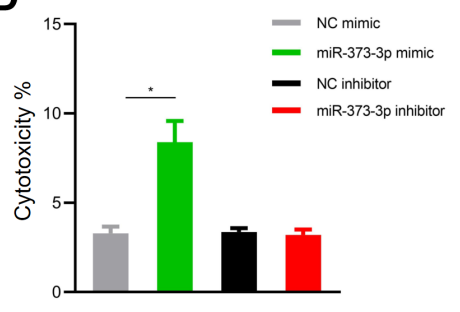

Figure 1. MicroRNA-373-3p inhibited the proliferation and reduced the viability of TC-1 cells

TC-1 cells were transfected with synthetic miR-373-3p mimic or miR-373-3p inhibitor. (A) Expression level of miR-373-3p in TC-1 cells after indicated transfection was detected using qPCR. (B) The proliferation of TC-1 cells after indicated transfection was analyzed by CCK-8. (C) Numbers of TC-1 cells after indicated transfection were counted. (D) The viability of TC-1 cells was analyzed using LDH release assay after indicated transfection. Data represent one of two independent experiments. Data represent Mean \pm S.E.M. ${ }^{*} P<0.05,{ }^{* *} P<0.01$ and $* * * P<0.001$ 
A

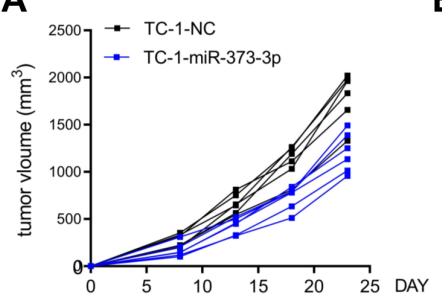

\section{C}

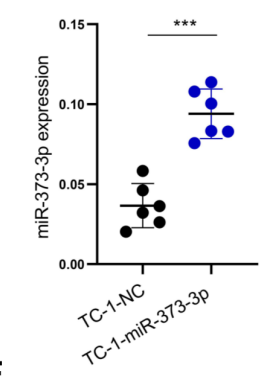

E



B

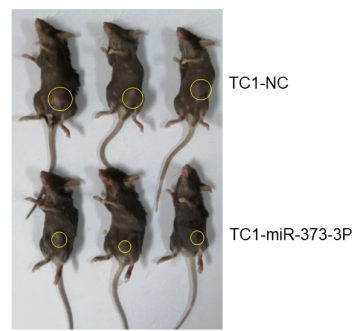

D

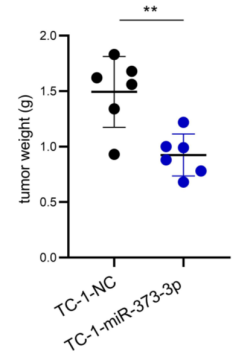

Figure 2. MicroRNA-373-3p inhibits tumor growth in TC-1 cervical cancer murine model

C57BL/6 mice were subcutaneously inoculated with stable TC-1 cells overexpressing miR-373-3p (A) Growth curve of TC-1 tumors was calculated. (B) Representative images of tumor-bearing mice. (C) The level of miR-373-3p in TC-1 tumors was detected using qPCR. (D) Mice were sacrificed on day 25, the weight of tumors was measured. (E) Survival curve of mice was calculated. Data are represented as one of two independent experiments. Data represent Mean \pm S.E.M. ${ }^{*} P<0.05$, ${ }^{* *} P<0.01$ and ${ }^{* * *} P<0.001$

\section{Western Blot}

Total protein was extracted from ice-cold lysis buffer. After being centrifuged for $5 \mathrm{~min}$ at $10000 \times \mathrm{g}$, equivalent amounts of protein $(20 \mu \mathrm{g})$ were subjected to $12 \%$ SDS-PAGE and transferred onto nitrocellulose membrane. Membranes were blocked for $60 \mathrm{~min}$ by phosphate-buffered saline. The membranes were then probed with primary antibodies against AKT1 (\#sc-5298, Santa Cruz, 1:1000), p-mTOR (\#2971, CST, 1:1000), mTOR (\#2972, CST, 1:1000) and GAPDH (\#5174, CST, $1: 1000)$, followed by the incubation with anti-mouse-IgG (\#A0258, Beyotime, 1:2000) or anti-rabbit-IgG secondary antibodies (\#A0239, Beyotime, 1:2000). Bands were visualized with the iBright Western Blot Imaging System (iBright CL1500, Thermo Fisher Scientific, Inc. USA).

\section{Cell transfection}

The pcDNA3.0-AKT1 plasmid (for AKT1 overexpression), sh-AKT1 plasmid (for AKT1 silence) and control plasmid were constructed by Genepharma (Co Ltd, China), $2 \mu \mathrm{g}$ plasmid was used for cell transfection. MiR-373-3p mimic (50 $\mathrm{nM})$, mimic control (50 $\mathrm{nM})$, miR-373-3p inhibitor (50 nM) and inhibitor control (50 nM) were synthesized by RiboBio (Guangzhou, China). Cervical cancer cells were transfected using Lipofectamine $^{\text {TM }} 2000$ Transfection Reagent (\#11668027, Invitrogen, Shanghai, China).

\section{Xenograft tumors model}

C57BL/ 6 mice (6-8 week, 6 mice/group) were subcutaneously injected with $10^{6}$ TC-1 cells in the groin. Mice were purchased from Vital River Laboratory Animal Technology Ltd. (Beijing, People's Republic of China). All animal experiments were approved by the Animal Ethics Committee of Nanjing University of Chinese Medicine. The tumor volume was calculated using the formula, $\mathrm{V}=(\mathrm{ab} 2) / 2$, where $\mathrm{a}$ is the long axis, and $\mathrm{b}$ is the short axis.

\section{Statistical analysis}

Unpaired Student's t-tests or log-rank test (for survival analysis) was used to assess the statistical differences between groups. Spearman's rank correlation test was performed to analyze the correlation between tumor volume and AKT1 expression. All statistical analyses were conducted by Prism Graphpad Software (Version 8.0). $P<0.05$ was considered statistically significant.

\section{RESULTS}

\section{MicroRNA-373-3p inhibits the proliferation of TC-1 cells}

To investigate the biological functions of miR-373$3 \mathrm{p}$ in cervical cancer cells, the TC-1 cells were transfected with miR-373-3p mimic or miR-373-3p inhibitor. The transfection efficiency was confirmed by QPCR 
A

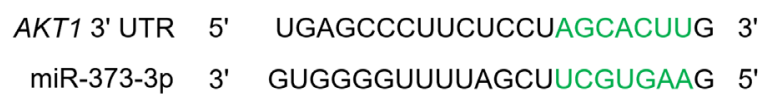

B

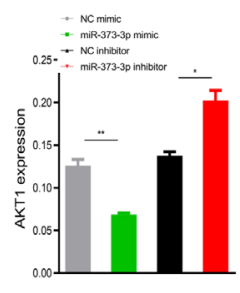

C


D

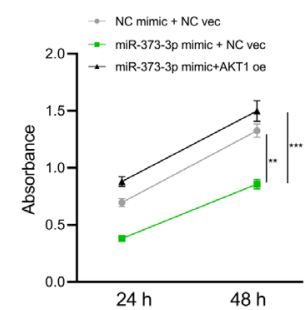

E

$\mathbf{F}$
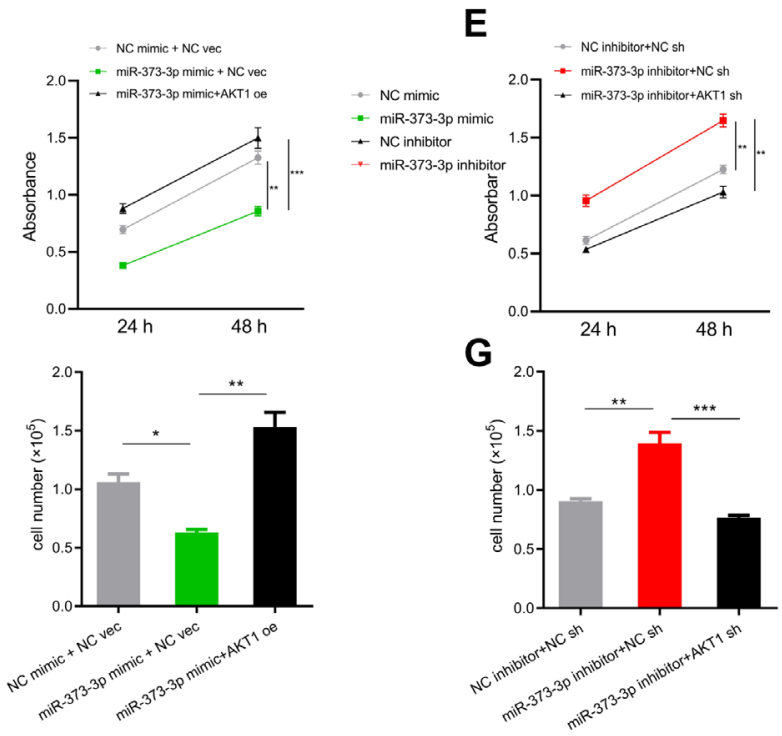

Figure 3. MicroRNA-373-3p inhibited cell proliferation by targeting AKT1 in TC-1 cells

TC-1 cells were transfected with synthetic miR-373-3p mimic or miR-373-3p inhibitor. (A) Schematic representation of potential binding site for mouse miR-373-3p in 3'-UTR region of AKT1. (B) The TC-1 cells were transfected with synthetic miR-373-3p mimic or antisense miR-373-3p inhibitor. The mRNA level of AKT1 in TC-1 cells after indicated transfection was detected using qPCR. (C) The protein levels of AKT1, p-mTOR, and mTOR in TC-1 cells were analyzed by Western blot after indicated transfection. Representative blots (left) and densitometric analysis (right) were shown. (D) TC-1 cells were co-transfected with AKT1 overexpression plasmids and miR-373-3p mimic or mimics control as indicated. The proliferation of TC-1 cells after indicated transfection was analyzed by CCK-8. (E) Numbers of TC-1 cells after indicated transfection in Fig. 2D were counted. (F) TC-1 cells were co-transfected with AKT1 knockdown plasmids and miR-373-3p inhibitor or negative control as indicated. The proliferation of TC-1 cells after indicated transfection was analyzed by CCK-8. (G) Numbers of TC-1 cells after indicated transfection in Figure 2F were counted. Data represent one of two independent experiments. Data represent Mean \pm S.E.M. ${ }^{*} P<0.05,{ }^{* *} P<0.01$ and ${ }^{* * *} P<0.001$

(Fig. 1A). As shown in Fig. 1B, compared with control mimic, the ectopic expression of miR-373-3p significantly inhibited the proliferation of TC-1 cells as evidenced by CCK-8 assay. In contrast, the inhibition of miR-373$3 \mathrm{p}$ enhanced the proliferation of TC-1 cells. Consistently, the number of TC-1 cells was decreased by miR373-3p overexpression and increased by miR-373-3p inhibitor (Fig. 1C). We also analyzed the viability of TC-1 cells after transfection with miR-373-3p mimic or inhibitor using LDH release assay. As shown in Figure 1D, increased percentage of dead TC-1 cells was found in miR-373-3p overexpression group. However, miR-373$3 p$ inhibitor failed to decrease the percentage of dead TC-1 cells compared with control inhibitor (Fig. 1D). Therefore, miR-373-3p can induce the growth inhibition and cytotoxicity in cervical cancer cells.

\section{MicroRNA-373-3p inhibits tumor growth in murine cervical cancer model}

To further investigate if miR-373-3p can also inhibit cervical cancer growth in vivo, we generated TC-1 cells with stable miR-373-3p overexpression (TC-1-miR-373-
$3 p)$ and inoculated these cells subcutaneously into mice. By monitoring tumor volume, we found that miR-373$3 p$ overexpression markedly suppressed the growth of cervical cancer cells in mice (Fig. 2A, B). By performing QPCR, we verified the successful overexpression of miR-373-3p in TC-1-miR-373-3p tumor tissues (Fig. 2C). In agreement, when the mice were sacrificed on day 25 , tumor weight from TC-1-miR-373-3p-inoculated mice was significantly reduced when compared to those from TC-1-NC-inoculated mice (Fig. 2D). In addition, miR373-3p overexpression significantly improved the survival of TC-1-bearing mice (Fig. 2E). Therefore, miR-373-3p also suppresses the in vivo growth of cervical cancer cells.

\section{MicroRNA-373-3p targets and downregulates AKT1 in cervical cancer cells}

Next, we investigated the target gene of miR-373-3p in cervical cancer cells. By performing target prediction algorithms using TargetScan base (www.targetscan.org), we identified a potential binding site for miR-373-3p in the 3'-UTR region of $A K T 1$ gene (Fig. $3 \mathrm{~A}$ ). To verify this prediction, The TC-1 cells were transfected with 
A

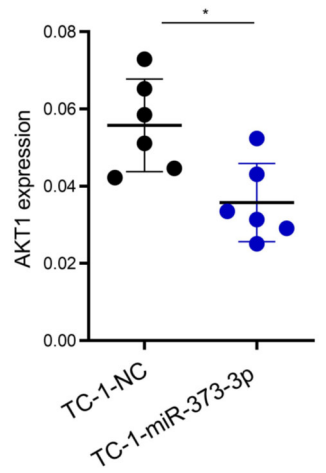

B

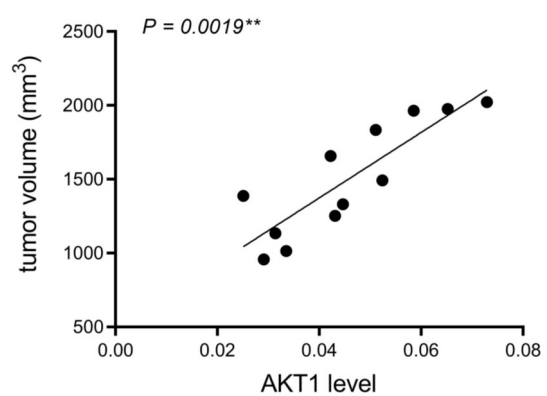

Figure 4. MiR-373-3p downregulated AKT1 in cervical tumor tissues

(A) The mRNA level of AKT1 in in TC-1 tumors was detected using GPCR. (B) Correlation between the tumor volume and AKT1 expression was analyzed by Spearman's rank correlation test

miR-373-3p mimic or miR-373-3p inhibitor. As shown in Fig. 3B and C, ectopic expression of miR-373-3p significantly decreased both the mRNA and AKT1 protein levels in TC-1 cells. In contrast, both the mRNA and protein levels of $A K T 1$ were increased by miR-373-3p inhibitor in TC-1 cells. Consistently, the phosphorylation of mTOR, the downstream effector of AKT1, was reduced by miR-373-3p overexpression, while its phosphorylation was enhanced by miR-373-3p inhibition (Fig. 3C).

To further validate whether $A K T 1$ is a functional target of miR-373-3p, TC-1 cells were co-transfected with AKT1 overexpressing plasmid and miR-373-3p mimic. As expected, miR-373-3p mimic markedly inhibited the proliferation of TC-1 cells, whereas AKT1 overexpression rescued the proliferation of TC-1 cells (Fig. 3D). Similar results were found in the number of TC-1 cells (Fig. 3F). On the other hand, TC-1 cells were co-transfected with sh-AKT1 and miR-373-3p inhibitor. MiR$373-3 p$ inhibition markedly promoted the proliferation of TC-1 cells, while the co-transfection of sh-AKT1 inhibited the proliferation of TC-1 cells (Fig. 3E). Similar results were found in number of TC-1 cells (Fig. 3G). These findings suggest that miR-373-3p suppresses TC-1 cell growth via downregulating AKT1 expression.

\section{MiR-373-3p downregulates AKT1 expression in cervical cancer tissues}

We then evaluated the $A K T 1$-targeting role of miR$373-3 p$ in tumor-bearing mice. To this end, we collected tumor tissues from TC-1-bearing mice. QPCR results showed that the expression of AKT1 mRNA in tumor tissues was significantly reduced in mice inoculated with TC-1-miR-373-3p than that in control mice (Fig. 4A).
Importantly, the mRNA level of AKT1 showed a significantly negative correlation with $\mathrm{miR}-373-3 \mathrm{p}$ in tumor tissues (Fig. 4B), indicating that miR-373-3p inhibits the in vivo growth of cervical cancer cells via targeting $A K T$ 1.

\section{MiR-373-3p inhibits cell proliferation by targeting AKT1} in human cervical cancer cells

Considering that the binding site for miR-373-3p in 3'-UTR region of $A K T 1$ is highly conserved between human and mouse (Fig. 5A), we then looked at the effect of miR-373-3p on two human cervical cancer cell lines. In HeLa cells, the ectopic expression of miR-373$3 \mathrm{p}$ significantly inhibited the cell proliferation, whereas inhibition of miR-373-3p enhanced the cell proliferation (Fig. 5B). Besides, ectopic expression of miR-373-3p significantly decreased the mRNA level of AKT1, while the miR-373-3p inhibitor increased the mRNA level of AKT1 (Fig. 5C). Similarly, in CaSki cells, the cell proliferation was inhibited by miR-373-3p mimic while enhanced by miR-373-3p inhibitor (Fig. 5D). Also, the expression of AKT1 mRNA was decreased by miR-373-3p overexpression while increased by miR-373-3p inhibition (Fig. 5E). Taken together, miR-373-3p serves as a tumor-suppressor miRNA by downregulating AKT1 in cervical cancer.

\section{DISCUSSION}

Cervical cancer caused approximately 265700 deaths worldwide in 2018 and poses a great threat to women's health (Bray et al., 2018). Despite the decreasing incidence and mortality rate of cervical cancer in recent years owing to the improved treatment approach, the early diagnosis of cervical cancer is still challenging due to the lack of effective biomarkers. The overall 5-year survival rate of cervical cancer is at about 40\% (Zhao et al., 2012), and the prognosis is still very poor (Marth et al., 2018). MicroRNAs have been widely reported to participate in regulating the progression of cervical cancer, so we aimed to identify new cervical cancer-associated microRNA in the present work.

MiR-373 is involved in the occurrence and development of many kinds of tumors, such as neuroblastoma (Yuan et al., 2019), oral squamous cell carcinoma (Zhang et al., 2019), lung Adenocarcinoma (Wu et al., 2015), colorectal cancer (Wang et al., 2018), gastric cancer (Shi et al., 2018), and pancreatic cancer (Hua et al., 2017). The relationship between miR-373 and CSCC is unclear. In this study, we revealed miR-373-3p as a new molecule inhibiting the growth of cervical cancer. It can suppress the proliferation and promote apoptosis of CSCC cells. Further research showed that as a tumor inhibitor, miR373-3p inhibited the growth of cervical cancer by targeting AKT1 gene. Overexpression of miR-373-3p inhibited AKT1 gene and protein expression, suggesting that miR373-3p promotes AKT1 signaling in cervical cancer cells. Phosphoinositide 3-Kinase (PI3K) - Protein Kinase B $(\mathrm{AKT})$ signaling pathway is frequently deregulated in human cancer, including cervical cancer. Activated AKT1 protein modulates the cascade expression of its downstream target genes, triggering a series of oncogenic programs that affect cell proliferation, differentiation, apoptosis, invasion, and migration. It has been found that AKT1 is activated in lung cancer, breast cancer, ovarian cancer, cervical cancer, and other tumors, and is involved in the important process of tumorigenesis and development, such as cell adhesion, movement, invasion, metastasis and so on. Aberrant activation of the PI3K-AKT1 


\section{A}

AKT13' UTR 5' UGAUCUCUCCACGGUAGCACUUG 3'

miR-373-3p 3' GUGGGGUUUUAGUUCGUGAAG 5'

B

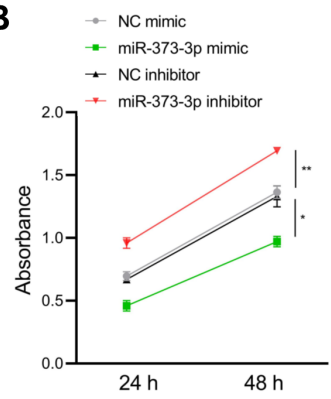

D

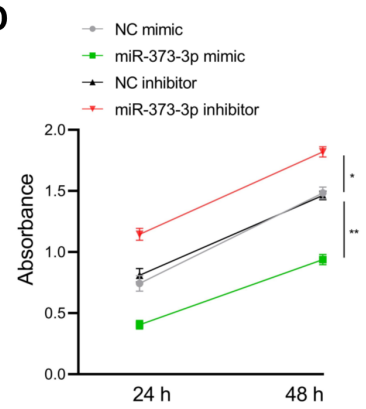

C $\quad-$ No mimic

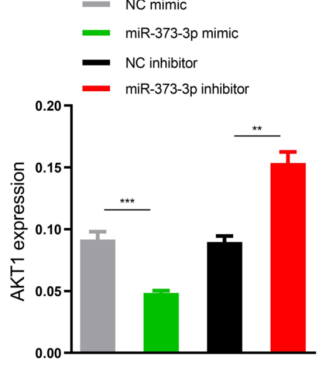

E

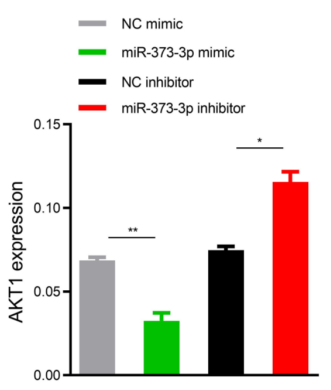

Figure 5. MicroRNA-373-3p inhibited cell proliferation by targeting AKT1 in human cervical cancer cells

(A) Schematic representation of potential binding site for human miR-373-3p in 3'-UTR region of AKT1. (B-E) HeLa cells (B, C) and CaSki cells (D, E) were transfected with synthetic miR-373-3p mimic or antisense miR-373-3p inhibitor. Cell proliferation was analyzed by CCK-8 (B, D). The level of $A K T 1$ in cervical cancer cells was analyzed by qPCR (C, E). ${ }^{*} P<0.05,{ }^{* *} P<0.01$ and ${ }^{* * *} P<0.001$.

signaling contributes to cervical cancer cell proliferation, Epithelial-Mesenchymal Transition (EMT), metastasis, survival, and angiogenesis (Shi et al., 2019).

Bioinformatics analysis suggested that $A K T 1$ may be a target of miR-373-3p. One study showed that the upregulation of miR-373 depleted its target gene AKT1 mRNA level, which led to the inhibition of AKTmTOR-S6K signaling pathway in hepatocytes that was critical in the development of hepatosteatosis ( $\mathrm{Li}$ et al., 2018). Another study showed that miR-373 promotes growth and cellular invasion in osteosarcoma cells by activating the PI3K/AKT-Rac1-JNK pathway (Liu et al., 2017). In this study, we for the first time compared the expression levels of AKT1 and miR-373-3p in CSC tissues vs. adjacent tissues. Furthermore, using human CSC cell line TC-1, the tumor-inhibiting effect of miR-373-3p in TC-1 was recovered by overexpression of AKT1, indicating the interaction between miRNA-373 and AKT1, but the in-depth mechanisms require further exploration.

However, there are still some unresolved questions, such as whether miR-373-3p has other targets in cervical cancer cells, what is the role of miR-373-3p in other tumors and whether it can inhibit other tumor progression by targeting $A K T 1$. Our research provides a mechanistic explanation for the increased proliferation in low miR373-3p expressing cervical cancer patients. In conclusion, we demonstrated that miR-373-3p is decreased in cervical cancer, and it may be a potential prognostic biomarker of cervical cancer. Inhibition of AKT1 may improve the treatment effect of cervical cancer. MiR-373-3p tar- geting $A K T 1$ is a potential novel molecular target for cervical cancer therapy.

\section{Acknowledgement}

Not Applicable

\section{Competing interests}

The authors state that there are no conflicts of interest to disclose.

\section{Availability of data and materials}

All data generated or analyzed during this study are included in this published article.

\section{Authors' contributions}

MMY and GJW conceived and designed the experiments, KHW, SLX and LLJ analyzed and interpreted the results of the experiments, QRL, AWX and GPY performed the experiments.

\section{Ethics approval and consent to participate}

Not Applicable

\section{Patient consent for publication}

Not Applicable 


\section{REFERENCES}

Bray F, Ferlay J, Soerjomataram I, Siegel RL, Torre LA, Jemal A (2018) Global cancer statistics 2018: GLOBOCAN estimates of incidence and mortality worldwide for 36 cancers in 185 countries. CA Cancer J Clin 68: 394-424. https://doi.org/10.3322/caac.21492

Croce CM, Calin GA (2005) miRNAs, cancer, and stem cell division. Cell 122: 6-7. https://doi.org/10.1016/j.cell.2005.06.036

Du P, Lai YH, Yao DS, Chen JY, Ding N (2019) Downregulation of microRNA-1246 inhibits tumor growth and promotes apoptosis of cervical cancer cells by targeting thrombospondin-2. Oncol Lett 18: 2491-2499. https://doi.org/10.3892/ol.2019.10571

Fan X, Xu S, Yang C (2018) miR-373-3p promotes lung adenocarcinoma cell proliferation via APP. Oncol Lett 15: 1046-1050. https:// doi.org/10.3892/ol.2017.7372

Hua Y, Chen H, Wang L, Wang F, Wang P, Ning Z, Li Y, Liu L, Chen Z, Meng Z (2017) Low serum miR-373 predicts poor prognosis in patients with pancreatic cancer. Cancer Biomark 20: 95-100. https://doi.org/10.3233/CBM-170231

Ji X, Guo H, Yin S, Du H (2019) miR-139-5p functions as a tumor suppressor in cervical cancer by targeting TCF4 and inhibiting Wnt/ beta-catenin signaling. Onco Targets Ther 12: 7739-7748. https://doi. org/10.2147/O'T'T.S215796

Li CH, Tang SC, Wong CH, Wang Y, Jiang JD, Chen Y (2018) Berberine induces miR-373 expression in hepatocytes to inactivate hepatic steatosis associated AKT-S6 kinase pathway. Eur I Pharmacol 825: 107-118. https://doi.org/10.1016/j.ejphar.2018.02.035

Liu L, Wang YL, Wang JF (2012) Differential expression of miR-21, miR-126, miR-143, miR-373 in normal cervical tissue, cervical cancer tissue and Hela cell. Sichuan Da Xue Xue Bao Yi Xue Ban 43: 536-539

Liu Y, Cheng Z, Pan F, Yan W (2017) MicroRNA-373 Promotes growth and cellular invasion in osteosarcoma cells by activation of the PI3K/AKT-Rac1-JNK pathway: the potential role in spinal osteosarcoma. Oncol Res 25: 989-999. https://doi.org/10.3727/096504 016X14813867762123

Lu R, Yang Z, Xu G, Yu S (2018) miR-338 modulates proliferation and autophagy by PI3K/AKT/mTOR signaling pathway in cervical cancer. Biomed Pharmacother 105: 633-644. https://doi.org/10.1016/j. biopha.2018.06.024

Manikandan J, Aarthi JJ, Kumar SD, Pushparaj PN (2008) Oncomirs: the potential role of non-coding microRNAs in understanding cancer. Bioinformation 2: 330-334. https://doi. org/10.6026/97320630002330
Marth C, Landoni F, Mahner S, McCormack M, Gonzalez-Martin A, Colombo N, ESMO Guidelines Committee (2018) Cervical cancer: ESMO Clinical Practice Guidelines for diagnosis, treatment and follow-up. Ann Oncol 29: iv262. https://doi.org/10.1093/annonc/ mdy160

Ngoma M, Autier P (2019) Cancer prevention: cervical cancer. Ecancermedicalscience 13: 952. https://doi.org/10.3332/ecancer.2019.952

Shi X, Wang J, Lei Y, Cong C, Tan D, Zhou X (2019) Research progress on the PI3K/AKT signaling pathway in gynecological cancer (Review). Mol Med Rep 19: 4529-4535. https://doi.org/10.3892/ mmr.2019.10121

Shi Y, Shi H, Zhang B, Yan Y, Han X, Jiang W, Qian H, Xu W (2018) miR-373 suppresses gastric cancer metastasis by downregulating vimentin. Mol Med Rep 17: 4027-4034. https://doi.org/10.3892/ mmr.2017.8291

Wang LQ, Yu P, Li B, Guo YH, Liang ZR, Zheng LL, Yang JH, Xu H, Liu S, Zheng LS, Zhou H, Qu LH (2018) miR-372 and miR-373 enhance the stemness of colorectal cancer cells by repressing differentiation signaling pathways. Mol Oncol 12: 1949-1964. https://doi. org/10.1002/1878-0261.12376

Wang S, Gao B, Yang H, Liu X, Wu X, Wang W (2019) MicroRNA-432 is downregulated in cervical cancer and directly targets FN1 to inhibit cell proliferation and invasion. Oncol Lett 18: 14751482. https://doi.org/10.3892/ol.2019.10403

Wei YQ, Jiao XL, Zhang SY, Xu Y, Li S, Kong BH (2019) MiR-9-5p could promote angiogenesis and radiosensitivity in cervical cancer by targeting SOCS5. Eur Rev Med Pharmacol Sci 23: 7314-7326. https://doi.org/10.26355/eurrev_201909_18837

Wu A, Li J, Wu K, Mo Y, Luo Y, Ye $\bar{H}$, Shen X, Li S, Liang Y, Liu M, Yang Z (2015) MiR-373-3p Promotes invasion and metastasis of lung adenocarcinoma cells. Zhongguo Fei Ai Za Zhi 18: 427-435. https://doi.org/10.3779/j.issn.1009-3419.2015.07.07

Yuan XL, Wen FQ, Chen XW, Jiang XP, Liu SX (2019) miR-373 promotes neuroblastoma cell proliferation, migration, and invasion by targeting SRCIN1. Onco Targets Ther 12: 4927-4936. https://doi. org/10.2147/OT'T.S205582

Zhang XJ, Jin Y, Song JL, Deng F (2019) MiR-373 promotes proliferation and metastasis of oral squamous cell carcinoma by targeting SPOP. Eur Rev Med Pharmacol Sci 23: 5270-5276. https://doi. org/10.26355/eurrev_201906_18193

Zhao YB, Wang JH, Chen XX, Wu YZ, Wu Q (2012) Values of three different preoperative regimens in comprehensive treatment for young patients with stage Ib2 cervical cancer. Asian Pac J Cancer Prev 13: 1487-1489. https://doi.org/10.7314/apjcp.2012.13.4.1487 